majority showing relapses and having residual weakness. After an initial improvement with prednisone therapy, attempts to withdraw steroids were often unsuccessful and the addition of immunosuppressive medications was rarely of benefit. The few children who recovered completely had an antecedent illness of URI or tonsillitis. Of two patients with CIDP associated with MMR immunization, none recovered and one had severe residual weakness. CIDP is a previously unreported side effect of MMR immunization. Transverse myelitis following MMR vaccine was reviewed in Ped Neur Briefs Sept 1995;9:65.

\title{
CONGENITAL AND JUVENILE MYASTHENIA GRAVIS
}

The clinical features, course, and presence of acetylcholine receptor antibody (AChRAb) were reviewed in 25 congenital (CMG) and 30 juvenile (JMG) cases of myasthenia gravis seen at Hacettepe University, Department of Paediatric Neurology, Ankara, Turkey. The age range of onset showed overlap: birth to 4 years for CMG, and 1.5 to 15 years for JMG. Parental consanguinity was present in $15(60 \%)$ of CMG and only $3(30 \%)$ of JMG patients. Motor development was delayed in $9(36 \%)$ CMG infants and in $3(10 \%)$ JMG patients. Initial symptoms were ocular in equal frequency for CMG (44\%) and JMG (53\%). After 1 year follow-up, only 4 (16\%) CMG patients had ocular only involvement, $19(76 \%)$ having progressed to ocular and bulbar or generalized weakness. Symptoms were limited to ocular muscles in $47 \%$ of JMG patients after 1 year. Unlike CMG, JMG patients showed spontaneous remissions in 20\% and myasthenic crises in $33 \%$. Good response to anticholinesterase drugs was more frequent in JMG than CMG (63 versus $41 \%$ ). AChRAbs were present in 9 (34\%) of JMG patients, all were girls with a later disease onset ( $>11 \mathrm{yrs}$ ) than antibody-negative cases. Pure ocular forms of MG were more often seronegative. None of the antibody-positive cases were in remission. The response to treatment was not significantly different between seropositive and negative cases. (Anlar B et al. Myasthenia gravis in childhood. Acta Paediatr July 1996;85:838-842). (Respond: Dr B Anlar, Hacettepe University, Department of Paediatric Neurology, Ankara 06100, Turkey).

COMMENT. In this series of childhood onset myasthenic patients, the proportion of congenital cases was much larger than previously reported. Facial muscle involvement and malformation often described in congenital cases was not alluded to in the above report. (see Progress in Pediatric Neurology I and II, PNB Publ, 1991 \& 1994). Family and developmental histories, severity and distribution of weakness, and response to therapy are supportive criteria for the differentiation of congenital and juvenile cases.

\section{GENETICS OF FACIOSCAPULOHUMERAL DYSTROPHY}

The relationship of phenotype to genotype in a clinically and genetically well defined population of 157 affected patients and 62 kindreds with facioscapulohumeral muscular dystrophy (FSHD) was examined at the University of Rochester School of Medicine, NY, and Ohio State University, Columbus, $\mathrm{OH}$. Using isometric myometry scores to quantify disease severity, a significant correlation between disease severity and the size of the 4q35associated deletion was evident, and the offspring were more severely affected than their parents. This generation effect and presence of anticipation in FSHD suggests a possible underlying dynamic mutation and an unstable repeat element within the region of the $4 \mathrm{q} 35$ deletion. (Tawil $\mathrm{R}$ et al. Evidence for anticipation and association of deletion size with severity in 
facioscapulohumeral muscular dystrophy. Ann Neurol June 1996;39:744-748). (Respond: Dr Tawil, University of Rochester, Department of Neurology, Box 673, 601 Elmwood Avenue, Rochester, NY 14642).

COMMENT. These findings have important significance in the genetic counselling of patients with FSHD. No differences in severity of disease were noted between paternally and maternally inherited FSHD, but a reduction in reproductive fitness in male compared to female patients was an unexpected finding.

FSHD with chromosome 9p deletion is reported in a 31-year-old man who also had congenital anomalies and mental retardation studied at Oita Medical University, Hasama-machi Oita 879-55, Japan. (Ueyama H et al. Neurology Feb 1996;46:566-569). A translocation between chromosome 4q and 9 p was not detected. The FSHD in this patient was probably not attributable to the $9 p$ deletion syndrome, which consists of the following: mental retardation, trigonocephaly, high-arched eyebrows, micrognathia, wide-spaced nipples, kyphosis, and inguinal hernias.

\section{ATTENTION DEFICIT AND LEARNING DISORDERS}

\section{QUANTITATIVE MRI CHANGES IN ADHD}

Anatomic brain MRIs for 57 boys with ADHD and 55 healthy matched controls, aged 5 to 18 years, were compared at the National Institute of Mental Health, Bethesda, MD. ADHD subjects had a $4.7 \%$ smaller total cerebral volume, a significant loss of normal right>left asymmetry in the caudate nucleus, smaller right globus pallidus, smaller right anterior frontal region, smaller cerebellum, and reversal of normal $(\mathrm{L}>\mathrm{R})$ lateral ventricular asymmetry. Whereas ventricular volume increased significantly with age for normal subjects, no age-related changes were found in ADHD subjects. Within the ADHD group, Full-Scale WISC-R IQ score correlated with total cerebral volume. Decreased normal caudate asymmetry was associated with increasing perinatal risk only in the ADHD boys. (Castellanos FX, Rapoport JL et al. Quantitative brain magnetic resonance imaging in attention-deficit hyperactivity disorder. Arch Gen Psychiatry July 1996;53:607-616). (Reprints: F. Xavier Castellanos MD, Child Psychiatry Branch, National Institute of Mental Health, Building 10, Room 6N240, 10 Center Dr, MSC 1600, Bethesda, MD 20892).

COMMENT. Evidence that a lack of normal asymmetry of regional brain structures is involved in the pathophysiology of ADHD is further supported by this study. Decreased volume of the prefrontal cortex, caudate nucleus, and globus pallidus on the right side point to a dysfunction of right-sided prefrontal-striatal systems in ADHD. A decrease in size of the splenium of the corpus callosum, previously reported in ADHD children (see Ped Neur Briefs July 1994;8:55), was not observed.

\section{ACUTE BASAL GANGLIA ENLARGEMENT WITH OBSESSIVE- COMPULSIVE DISORDER, TICS, AND STREP INFECTION}

A 12-year-old boy with an acute exacerbation of obsessive-compulsive disorder (OCD) symptoms and tics following a Group A B-hemolytic streptococcal (GABHS) throat infection is reported from the National Institute of Mental Health, Bethesda, MD. Family history included Sydenham's chorea in a maternal grandfather, OCD in the mother and paternal aunt, and Tourette's 